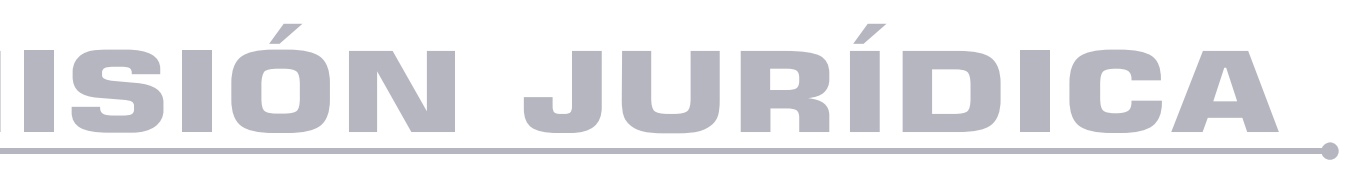

\title{
Revolución digital, economía creativa y economía social y solidaria: conexiones y contribuciones
}

Digital revolution, creative economy and social and solidarity economy: connections and contributions

Autor: Daniel Francisco Nagao Menezes

DOI: https://doi.org/10.25058/1794600X.1918

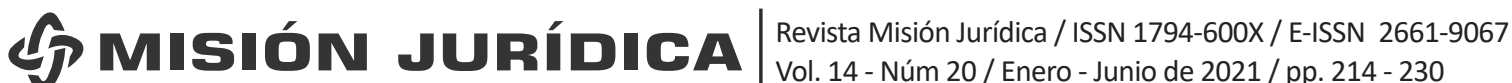




\title{
REVOLUCIÓN DIGITAL, ECONOMÍA CREATIVA Y ECONOMÍA SOCIAL Y SOLIDARIA: CONEXIONES Y CONTRIBUCIONES*
}

\author{
Digital revolution, creative economy and social and solidarity \\ economy: connections and contributions \\ Revolução digital, economia criativa e economia social e \\ solidária: conexões e contribuições
}

Daniel Francisco Nagao Menezes ${ }^{\mathrm{a}}$

Fecha de recepción: 13 de julio de 2020

Fecha de revisión: 15 de julio de 2020

Fecha de aceptación: 22 de marzo de 2021

DOI: https://doi.org/10.25058/1794600X.1918

Para citar este artículo:

Menezes Nagao, D. F. (2021). Revolución digital, economía creativa y economía social y solidaria: conexiones y contribuciones. Revista Misión Jurídica, 14, (20), 214-230.

\section{RESUMEN}

El artículo analiza la contribución de la Economía creativa, en la perspectiva de la Economía Social y Solidaria (ESS), en tiempos de Revolución Digital, en un contexto en el que aún prevalecen altos y crecientes niveles de desigualdad socioeconómica y de exclusión social. La metodología empleada es la hipotética deductiva con el uso de la revisión bibliográfica. La hipótesis explorada es la existencia de una aproximación entre economía creativa y ESS permitiendo a la economía creativa la utilización de las estructuras jurídicas que ésta posee. El punto común que permite

\footnotetext{
*Artículo resultante del Proyecto de Investigación 402885/2018-0 - (Re) construyendo el Derecho Cooperativo Latinoamericano - Creando una matriz teórica e institucional para América Latina - financiado y apoyado por el CNPQ en la Edicto CNPQ / SESCOOP No 07/2018.

a. Graduación en Derecho (PUC-Campinas), Especializaciones en Derecho Constitucional y Derecho Procesal Civil (PUC-Campinas), en Didáctica y Práctica Pedagógica en la Educación Superior (Centro Universitário Padre Anchieta), Master y Doctor en Derecho Político y Económico (Universidade Presbiteriana Mackenzie), Post-Doctor en Derecho (USP). Post-Doctor en Economía (UNESP-Araraquara). Profesor del Programa de Posgrado en Derecho Político y Económico de la Facultad de Derecho de la Universidade Presbiteriana Mackenzie. Profesor colaborador de la Maestría en Economía Social de la Universidad Autónoma de Guerrero (Acapulco, México). Miembro de CIRIEC-Brasil.
} 
dicha aproximación es la base colaborativa entre los tipos de emprendimientos y a la vez que sean clasificados como sociales y solidarios.

\section{PALABRAS CLAVE}

Tecnología; Economía creativa; Economía colaborativa; Economía Social y Solidaria; Bien común.

\section{ABSTRACT}

The objective of the article is to discussanalyzes the contribution of the Creative Economy, in the perspective of the Social and Solidarity Economy (ESS), in times of Digital Revolution, but in a context where high and increasing levels of socioeconomic inequality and social exclusion still prevail. The methodology employedused is the hypothetical deductive with the use of the bibliographic review. The hypothesis exploresd in conclusion is that the existing alternative is the approximation between creative economy and ESS, allowing the creative economy to use the legal structures that it possesses. The common point that allows thesuch approximation between the two ways of doing economy is the collaborative basis between the types of ventures and allows them tothat at the same time can be classified as social and solidarity.

\section{KEYWORDS}

Technology; Creative economy; Collaborative economy; Social and Solidarity Economy; Common benefit.

\section{RESUMO}

0 artigo analisa a contribuição da Economia Criativa, na perspetiva da Economia Social e Solidária (ESS), em tempos de Revolução Digital, num contexto em que ainda prevalecem elevados e crescentes níveis de desigualdade socioeconómica e exclusão social. A metodologia utilizada é a hipotética dedutiva com recurso à revisão bibliográfica. A hipótese explorada é a existência de uma aproximação entre a economia criativa e a ESS, permitindo à economia criativa utilizar as estruturas jurídicas de que dispõe. 0 ponto comum que permite esta abordagem é a base colaborativa entre os tipos de empresas e ao mesmo tempo que são classificadas como sociais e solidárias.

\section{PALAVRAS CHAVE}

Tecnologia; Economia criativa; Economia colaborativa; Economia Social e Solidária; Bem comum.

\section{INTRODUCCIÓN}

La economía mundial ha experimentado, en las últimas décadas, una transformación profunda e irreversible con el surgimiento de nuevos sectores económicos adaptados a la nueva realidad tecnológica. Estos cambios, junto a los que se han dado en la orientación de las políticas económicas nacionales e internacionales, llevan a la necesidad de adaptación y creación de nuevas herramientas legales capaces de apoyar los nuevos arreglos productivos.

La llamada economía creativa encaja en uno de estos nuevos acuerdos de producción, y es un término, aún no definido con precisión, que designa modelos de negocios o de gestión que se basan en actividades, productos o servicios desarrollados e implementados desde el conocimiento y la creatividad o el capital intelectual de los individuos. A diferencia de la economía tradicional, la industria, la agricultura y el comercio, la economía creativa se centra en el potencial individual o colectivo de producir bienes y servicios creativos, ya sean tangibles o intangibles, intelectuales y artísticos, con contenido creativo y valor económico, para el surgimiento de una nueva forma de producción y distribución de la riqueza. Las actividades "creativas" se ubican, principalmente, en sectores como cultura, moda, diseño, música y artesanías. Otra parte importante se encuentra en el sector de la tecnología y la innovación, como el desarrollo de software, juegos electrónicos y teléfonos móviles. También se incluyen actividades de televisión, radio, cine y fotografía, así como la expansión de los usos de Internet.

Además de los beneficios económicos que vienen con la incorporación de una "nueva" forma de producción y distribución de riqueza, la economía creativa también contribuye significativamente al desarrollo social, a diferencia de la economía tradicional. Esto por lo tanto, teniendo en cuenta que la economía creativa valora el espíritu empresarial y la autogestión, cuya consecuencia puede ser el crecimiento inclusivo y sostenible y la diversificación de los mecanismos de generación y distribución de la 
riqueza. Sin embargo, desde esta perspectiva, las nuevas estructuras de la economía creativa solo serán efectivas si tuvieran el elemento clave de la colaboración. Dentro de la economía, la rama que realiza actividades económicas basadas en la colaboración es la Economía Social y Solidaria (ESS). Así, la economía creativa tiene en principio la formación de redes colaborativas, y los principios de la ESS son consistentes con este desideratum. Con el surgimiento de economías plurales, se percibe cada vez más la presencia de redes productivas que cooperan como condición para su desarrollo. En otras palabras, la ESS es cada vez más importante hoy, dada la crisis económica mundial y las transformaciones tecnológicas, ya que los resultados requerirán, entre otros aspectos, un modelo de desarrollo más inclusivo y sostenible (Morais, L.; Dash, A., y Bacic, M., 2016).

En este sentido, aquí se pretende discutir el aporte de la economía creativa, desde la perspectiva de la ESS, en tiempos de Revolución Digital, pero en un contexto donde prevalecen altos y crecientes niveles de desigualdad socioeconómica y exclusión social. Inicialmente, el artículo presentará una breve discusión teórico-conceptual sobre los términos ESS, economía colaborativa, economía creativa, redes colaborativas, etc. Luego, se presentan algunos ejemplos prácticos, así como algunas reflexiones acerca de la necesidad de adaptación y creación de nuevas herramientas legales capaces de soportar estos nuevos arreglos productivos.

La economía creativa puede entenderse como una nueva forma de "ahorrar", ya que es una nueva forma de producción y distribución de riqueza. En esta dirección, la economía creativa parece ser, de hecho, un potencial importante para el desarrollo socioeconómico, aprovechando un momento de transición de los paradigmas globales a la reorganización de recursos y la distribución de beneficios económicos.

En esta perspectiva, la ESS se erige como un instrumento para reflexionar y actuar sobre este nuevo escenario y sus desafíos inherentes. La ESS incluye elementos que combinan la generación de trabajo e ingresos con la participación social y comunitaria, así como la preservación del medio ambiente, la inserción socioeconómica de los involucrados y la preocupación por el entorno territorial. Es decir, abarca factores económicos, pero también sociales y ambientales.

Se encuentran varios obstáculos, como la falta de una definición conceptual precisa de lo que comprende la economía creativa y, cómo funciona. La ausencia de definiciones tiene consecuencias para las estructuras legales que no crean normas legales capaces de permitir el desarrollo completo de empresas basadas en la economía creativa. La respuesta es la posibilidad de utilizar las estructuras legales y de gestión de la ESS, que tiene una de las características centrales de la adaptación a las estructuras legales adversas.

La hipótesis que se desarrollará en este trabajo es que la economía creativa tiene sus bases económicas en la Economía Colaborativa, que propone el uso más racional de los bienes y servicios escasos, lo que permite la potencialización de su uso y el mayor alcance de estos bienes y servicios que llegan a un número determinado de personas.

A su vez, considerando el carácter racionalizador y expansionista de la economía colaborativa, puede clasificarse como una rama de la economía social y solidaria. En esta línea de razonamiento, al considerar que la economía creativa tiene los mismos supuestos y características de la economía colaborativa, se puede afirmar, aunque provisionalmente, que la economía creativa es parte de la Economía Social y Solidaria.

Por lo tanto, la regulación legal de la economía creativa debe partir de los principios jurídicos de la ESS, bajo la pena de hacerlo bajo otros principios, para prevenir el desarrollo de la economía creativa. Entendida aquí en su sentido más amplio, está creando precedentes importantes a diferencia de las áreas económicas tradicionales y menos dinámicas, especialmente al interpretar de forma creativa la aplicación del conocimiento de una manera innovadora, adoptando tecnologías y nuevos modelos de negocios y cooperación, pensados a través de planes internacionales Utilizando la tecnología para comunicarse con clientes y proveedores.

Vale la pena señalar que el valor real de la economía creativa no solo reside en el valor económico, o incluso en su impacto social y cultural más amplio, sino que también representa 
un nuevo modelo de gestión de la riqueza y producción. Así como las grandes fábricas de los siglos XIX y XX configuraron la economía, los estados nacionales, los sistemas educativos $y$, especialmente, la organización de la sociedad, la economía creativa apunta a establecer en este siglo la dirección de la economía mundial.

La revolución tecnológica, iniciada a fines del siglo pasado, está directamente relacionada con la expansión de la economía creativa al incrementar la producción cultural y sus formas de valorización y preservación, tanto cuantitativa como cualitativamente. Por otro lado, renueva la agenda política de cultura y conocimiento y la importancia de estos dentro de la sociedad. La búsqueda desenfrenada de nuevos paradigmas de la producción económica postfordista, la incapacidad de responder a las formas tradicionales de producción y la composición del marco adverso que la economía creativa busca consolidar.

El artículo está dividido en cinco apartados (contextualización de la Revolución Digital, balizamiento teórico-conceptual y, economía creativa y la Economía Social y Solidaria) con un corte geográfico iberoamericano $\mathrm{y}$, un corte temporal a partir de los años 2000 en adelante, un momento histórico que consolida la economía creativa en la región.

\section{REVOLUCIÓN DIGITAL DEL SIGLO XXI: BREVE CONTEXTUALIZACIÓN}

Se vive una nueva era, marcada por profundos e irreversibles cambios tecnológicos cuyos desdoblamientos todavía son desconocidos. Esta nueva era se caracteriza por avances que van hacia la inteligencia artificial, "internet de las cosas", big data, computación en nube, vehículos autónomos, robótica avanzada, etc. No es casual que muchos investigadores en todo el mundo se inclinan sobre la cuestión referente al alcance de este cambio tecnológico, actualmente en curso.

Los avances en robótica, inteligencia artificial y aprendizaje de máquinas ya han iniciado una nueva era de automatización, donde las máquinas combinan o superan el desempeño humano en una serie de actividades de trabajo, incluyendo aquellas que exigen capacidades cognitivas. Estas nuevas tecnologías tienen por objeto mejorar significativamente los productos, los procesos, los métodos organizativos y los mercados, sobre la base de mejoras permanentes y crecientes en la automatización, así como en la capacidad de almacenamiento y transferencia de comunicaciones en general.

La digitalización de la producción -una de las tendencias de esta nueva era- conocida como "fabricación avanzada" o "Industria 4.0" está desempeñando un papel importante en la determinación no solo de la naturaleza de trabajo, sino también en su disponibilidad. Esto significa que las máquinas pueden implantarse en el lugar de trabajo a una escala mucho mayor que en el pasado y a un ritmo mucho más rápido, cambiando el proceso de producción de una manera que tiene consecuencias de largo alcance para la productividad, el empleo, la competencia, la distribución de ingresos, la realización del comercio, etc.

En esta perspectiva, avances en robótica, inteligencia artificial y aprendizaje de máquina están abriendo nuevos horizontes para automatización. Por lo tanto, estamos entrando en un momento en que las tecnologías están evolucionando más rápidamente que las habilidades y las organizaciones. Son ejemplos prácticos y ya existentes de esta nueva era: narrative science (software que puede escribir noticias); tecnologías financieras, como Fintech, que están entre las más rápidas tecnologías en crecimiento; Watson-IBM puede analizar en pocos segundos toda la literatura médica para producir un diagnóstico; MOOC (cursos en línea abiertos en masa); vehículos autónomos, ya que el siguiente paso en la estrategia de Uber es sustituir a los conductores vía inteligencia artificial, etc.

El reciente trabajo de Mc Kinsey (2017) analizó el potencial de automatización de la economía global, los actores que determinar el ritmo y la extensión de la adopción en el lugar de trabajo $\mathrm{y}$, el impacto económico asociado a su potencial. Entre las constataciones de este estudio, se citan:

- La automatización de actividades permite a las empresas mejorar el rendimiento, reduciendo errores, mejorando la calidad y la velocidad y, en algunos casos, alcanzando resultados que van más allá de las capacidades humanas;

- La automatización contribuye a la productividad, como ha hecho históricamente. Con base en el modelado de escenario 
realizado, se estima que la automatización puede aumentar el crecimiento de la productividad globalmente de 0,8 a 1,4\% al año;

- Casi la mitad de las actividades ocupacionales mundiales tienen el potencial de ser automatizadas, total o parcialmente;

- Las actividades más susceptibles a la automatización involucra actividades físicas en ambientes altamente estructurados $y$ previsibles, así como la recolección y el procesamiento de datos. No entanto, percebese também que esta nova era trata-se de um fenômeno que não está restrito ao mundo empresarial e industrial, mas encontrase cada vez mais difundido em inúmeras dimensões da vida pessoal e cotidiana.

Nota: revisar lo resaltado en cuanto a lo que le precede

Uno de los aspectos más desafiantes de esta "sociedad informacional" es la rápida y abrumadora difusión de lo que se está convenciendo llamar "economía colaborativa" o "economía compartida" (sharing economy). Obviamente, esto se debe, en gran parte, a la interconectividad más fácil y más económica propiciada por los recursos tecnológicos disponibles, especialmente las nuevas tecnologías de información, lo que permitió superar el modo de comunicación unidireccional gracias a la práctica interactiva de auto comunicación de masa. Compartir a través de plataformas digitales permite importantes mejoras de eficiencia en la combinación de los factores de producción, la creación de un nivel más avanzado de funcionamiento sistémico, e impulsar los movimientos sociales en la red $\mathrm{y}$, las nuevas formas de intercambio económico y manifestación política (Lisboa, 2017).

La economía creativa se puede agrupar en cuatro áreas creativas principales: consumo (diseño, arquitectura, moda y publicidad y marketing), medios (editorial y audiovisual), cultura (patrimonio y artes, música, artes escénicas y expresiones culturales) y tecnología (I+D), Biotecnología y TIC) ( Firjan, 2019, p. 3).

Analizando las cifras de Brasil, en 2017 la economía creativa representó el 2,61\% del PIB, empleó a 837 mil trabajadores y el ingreso mensual promedio del trabajador brasileño fue de $\mathrm{R} \$ 2.777,00$ en 2017, el de los profesionales creativos, generalmente más calificados, fue 2,45 veces mayor y alcanzó R\$6.801,00 (p. 4).

Hoy, el impacto de la economía creativa en la actividad económica brasileña es extremadamente relevante. El número de nuevos empleos está creciendo: entre 2015 y 2017, se perdieron alrededor de 1,7 millones de empleos tradicionales en el país. En el mismo período, se crearon más de 25 mil nuevas vacantes para diez profesiones dentro de la economía creativa. La expectativa es que la economía creativa brasileña alcanzará los US\$ 43.7 mil millones para 2021 (p. 14).

Es una estrategia de desarrollo para las personas ausentes de los sistemas formales de trabajo y así organizarse. Según los datos del informe de la United Nations Conference Trade and Development (2018), el mercado mundial de productos creativos, a pesar de la crisis financiera de 2008 que afectó la creación y distribución de productos creativos, tuvo una tasa de crecimiento promedio superior a 7\% entre 2002 y 2015. El mercado mundial de productos creativos se duplicó de US\$208 mil millones en 2002 a 509 mil millones en 2015. Estas cifras confirman que las industrias creativas han sido uno de los sectores más dinámicos de la economía mundial, lo que hace que la economía creativa sea una de las principales estrategias de desarrollo de los países para el siglo XXI.

En este contexto, una cuestión fundamental reside en la indagación de: ¿será esta nueva era capaz de permitir, con tales instrumentos oriundos de la magnífica evolución tecnológica, de contribuir al enfrentamiento de la pobreza, de la desigualdad socioeconómica y de accesos a los bienes públicos esenciales?

Tema discutido en el último congreso del CIREC Internacional, celebrado en mayo de 2018, en la ciudad de Lieja, Bélgica. Como uno de los productos de este evento, que contó con la participación de más de 400 delegados de más de 30 países, se concibió la Declaración de Lieja (2018). En esta Declaración, existe el reconocimiento explícito de que:

Si las nuevas tecnologías digitales generan la esperanza de ver la implementación de soluciones de progreso para las 
poblaciones y de mejora de su bienestar, también suscitan temores de que todos estos avances tecnológicos basados en la inteligencia artificial acaben esclavizando a la humanidad en beneficio de algunos gigantes mundiales de la economía y de las finanzas. Las implicaciones de la tecnología digital en la organización de las sociedades y en las relaciones entre las personas son efectivamente tales que, más allá de las perspectivas positivas de mejoramiento de las condiciones de vida en nuestros territorios, también pueden entrañar peligros en términos de ética, democracia y aumento de las desigualdades.

En este sentido, hay intención de reflexionar sobre las potencialidades de esta nueva era, en contribuir con el enfrentamiento de problemas estructurales como la pobreza, la desigualdad de accesos, así como posibilidades de generación de trabajo y renta, se cree que entender las posibles conexiones de esta nueva era de la "economía creativa, compartida o colaborativa" con la economía social y solidaria constituye un pertinente y relevante desafío.

Incluso si se tiene en cuenta la provocación de autores como Lisboa (2017, p. 6) en la que:

Apesar de estarmos assistindo à ascensão espontânea de produção cooperativa mediadas pelas redes computacionais, há um profundo descaso por parte do movimento da economia solidária para com este fenômeno, e vice-versa. Como é sabido, o que comumente se denomina de economia solidária identifica modelos que vão além de simples empreendimentos econômicos, pois buscam novas formas de convivência coletiva geradoras de trabalho e renda a partir de relações de mutualidade e de reciprocidade, apoiadas na solidariedade e na equidade. Portanto, em face de evidentes afinidades, era de se esperar que o fenômeno da economia compartilhada cruzasse, fertilizasse e potencializasse ampla e significativamente o movimento da economia solidária. Todavia, isto não tem ocorrido. Em verdade, a reflexão e as redes da economia solidária não se abriram para esta dimensão das plataformas colaborativas.
Para el autor, indudablemente, el "cooperativismo de plataforma", como propone Scholz (2016), es una gran posibilidad para el desarrollo de emprendimientos de economía solidaria. En su visión, "si las certezas ideológicas son desnudas, si liberamos el concepto de economía solidaria de sus amarras, una identificación común, y un diálogo, podrán surgir entre estos dos grandes campos que incuban contemporáneamente otra economía".

Tema tratado también por Fonseca y Machado (2013), al analizar cómo los emprendimientos económicos solidarios (EES) utilizan los medios electrónicos para compartir conocimientos y tecnologías sociales, movilizar recursos y comunicarse con los miembros del movimiento en la región de Belo Horizonte, en el Estado de Minas Gerais. Según los autores, que parten de la idea de que la emergencia de las tecnologías de comunicación e información y la diseminación de la cultura derivada de su utilización, la cultura digital, originariamente representada por Internet, trajeron la expectativa de poder promover cambios sociales, económicos, culturales, extensivos para la sociedad como un todo. Sin embargo, es curioso verificar a partir de los datos de este estudio que no es la carencia material (de equipamientos y conexión) que impide el uso de Internet por los EES, pero si las trabas de cuño cultural, pues éstas implican la adopción de hábitos, actitudes y comportamientos de nuevo tipo.

En ese sentido, vale reproducir nuevamente otro trecho de la ya citada Declaración de Lieja (2018), donde:

En esta situación de aceleración temporal sin precedentes, y dados los riesgos de desviar lo que debería considerarse un progreso para las poblaciones, es esencial que los actores de la economía pública, social y cooperativa se apropien todos estos temas porque es esencial que esta extraordinaria promesa tecnológica que ofrece la tecnología digital se transforme en progreso económico, social, democrático y ambiental.

Esta afirmación encuentra respaldo en otros estudios que apuntan al hecho de que, frente a los cambios tecnológicos ya en curso y los impactos en la destrucción masiva de los empleos, además de los profundos cambios en la 
composición sectorial de las ocupaciones, una de las importantes actividades es la apuesta por lo social del sector; sector este que contempla una serie de actividades que pueden ser disponibles por la ESS (Peruffo, Schmidlechner, Contreras y Molinuevo, 2017).

A partir de estas colocaciones, aparecen innumerables preguntas. Entre ellas, la primera se relaciona con la sociología económica, al indagar cómo las nuevas plataformas digitales impactan la vida en sociedad, partiendo del supuesto de que hay cambios en las relaciones económicas. La segunda, se refiere a la comprensión de cómo estas nuevas relaciones económicas (y también sociales) organizarán la economía y las actividades de producción y consumo; situándose aquí, la tercera pregunta: cómo la Economía Social y Solidaria (ESS) se relaciona con esta nueva forma de hacer economía.

La ESS nace abajo de criterios cooperativistas, una concepción que toma muchos aspectos del cooperativismo, una economía solidaria que se concibe tanto del punto de vista macro como micro. Jaramillo (2005) define que por el lado macro, se habla de un sector en general y, por el micro, tiene una concepción entendida como una unidad asociativa, la cual es capaz de generar bienes y servicios regidos por unos principios de autogestión, ayuda mutua y solidaridad. Los principios fundamentales sobre los cuales se forja la economía solidaria son: la reconstrucción de comunidades, la autogestión, la ayuda mutua frente al progreso humano, la cooperación fundamente del trabajo común, la solidaridad y la participación democrática.

Considerando que la Economía Digital no tiene conceptos claros y legalmente precisos, hay un espacio político en disputa sobre la mejor manera de organizar actividades económicas digitales, creando un momento histórico favorable para que la economía solidaria discuta el espacio y las reglas de la organización económica digital.

Con el fin de reflexionar sobre estas cuestiones, los siguientes temas tratarán de bautizar teóricoconceptualmente los términos economía creativa y ESS para, posteriormente, intentar encontrar puntos de convergencia entre ambos mundos.

\section{EN BUSCA DE UN BALIZAMIENTO TEÓRICO-CONCEPTUAL}

2.1. Aspectos teórico-conceptuales acerca de la economía creativa (EC)

Antes de entrar en la discusión de las alteraciones de la economía creativa como parte de la ESS, es necesaria la comprensión de sus conceptos fundamentales, lo que comprende y cómo funciona en las economías de los países desarrollados y en desarrollo.

Al tratar de economía creativa, el primer concepto a ser enfrentado es la definición de creatividad. No existe un concepto preciso que englobe todas las dimensiones de esta. Para la psicología la creatividad ha sido ampliamente estudiada, no existe consenso sobre la cuestión de la creatividad como atributo humano o un proceso por el cual se crean ideas originales, generando así una duda epistemológica sobre el inicio del proceso de creación. La Organización de las Naciones Unidas (ONU), en su Informe (UNCTAD, 2018), distingue tres tipos de creatividad:

- criatividade artística envolve a imaginação e a capacidade de gerar ideias originais e novas maneiras de interpretar o mundo, expressas em texto, som e imagem;

- criatividade científica envolve curiosidade e disposição para experimentar e fazer novas conexões ao solucionar problemas; e

- criatividade econômica é um processo dinâmico que leva à inovação em tecnologia, práticas de negócio, marketing, etc., sendo intensamente relacionada à aquisição de vantagem competitiva na economia;

Otro concepto para discutir es la capacidad de la economía creativa para ser industrializable, es decir, pasar de una economía de alcance a una economía de escala, forma a la que se lo denomina Industria Creativa. Esta definición, a su vez, es una cuestión de considerable inconsistencia y divergencia en las discusiones académicas y también en la legislación, especialmente en relación al concepto paralelo de Industrias Culturales.

El punto de convergencia entre los conceptos es el resultado, los llamados "productos culturales", esto es vinculado con la noción de cultura, en su sentido antropológico o funcional. Ejemplificando: productos y servicios culturales 
tales como trabajos artísticos, performances musicales, literatura, películas, programas de televisión y videojuegos. El informe de la ONU (2013) que trae algunas características básicas de los productos culturales:

- sua produção demanda alguma contribuição da criatividade humana;

- eles são veículos de mensagens simbólicas para aqueles que os consomem, isto é, eles são mais do que simplesmente utilitários, na medida em que também servem a um propósito comunicativo mais amplo; e

- $\quad$ eles contêm, pelo menos, potencialmente, alguma propriedade intelectual que possa ser atribuída ao indivíduo ou grupo que esteja produzindo o produto ou serviço

Con ello, las industrias culturales se pueden clasificar como industrias que conjugan la creación, producción y comercialización de contenidos intangibles y culturales por naturaleza, pudiendo asumir la forma de productos y servicios y, protegidos por la legislación de propiedad intelectual.

Es importante destacar que este concepto de Industria de la Cultura sufrió una alteración en la XI Conferencia Ministerial de la UNCTAD, en 2004, pasando a usarse la "Industria Creativa". En este evento, la temática de la industria creativa fue introducida en la agenda económica y de desarrollo internacional, formando un Panel de Alto Nivel sobre Industrias Creativas y Desarrollo. El enfoque de la UNCTAD para las industrias creativas busca ampliar el concepto de creatividad, pasando de actividades que poseen componentes artísticos para "qualquer atividade econômica que produza produtos simbólicos intensamente dependentes da propriedade intelectual, visando o maior mercado possível" (UNCTAD, 2010).

En las recomendaciones derivadas del Panel de la UNCTAD, se crea la diferencia de actividades upstream (actividades culturales tradicionales, tales como artes escénicas o visuales) de actividades downstream (que poseen una proximidad mucho mayor con el mercado, como publicidad, editoriales o actividades relacionadas con los medios de comunicación), y señala que las actividades downstream derivan su valor comercial de los bajos costos de reproducción y fácil transferencia a otros ámbitos económicos.
La Conferencia de la UNCTAD, realizada en la ciudad de São Paulo, se desarrolló buscando aún ampliar el rol de definiciones técnicas, e influenciar políticas públicas, creando así una fuerte relación con el concepto de economía creativa. Para UNCTAD (2010) son cuatro los objetivos principales de la economía creativa:

- Reconciliar os objetivos culturais nacionais com as políticas comerciais tecnológicas e internacionais;

- Analisar e solucionar as assimetrias que estejam inibindo o crescimento das indústrias criativas nos países em desenvolvimento;

- Reforçar o chamado "nexo criativo" entre investimento, tecnologia, empreendedorismo e comércio; e

- Identificar respostas de políticas inovadoras para aprimorar a economia criativa a fim de gerar ganhos de desenvolvimento.

A partir de estas premisas se delimitan cuatro campos de actuación de la economía creativa, según el estudio de Araújo, Oliveira y Silva (2013, p. 18) son estas áreas:

Grupo 1 - Patrimônio: o patrimônio cultural é identificado como a origem de todas as formas de artes e a alma das indústrias culturais e criativas, o ponto de partida para esta classificação. É a herança que reúne aspectos culturais dos pontos de vista histórico, antropológico, étnico, estético e social, e influencia a criatividade dando origem a uma série de bens e serviços do patrimônio, bem como atividades culturais. Este grupo é, portanto, dividido em dois subgrupos: a) expressões culturais tradicionais: artesanato, festivais e celebrações; e b) locais culturais: sítios arqueológicos, museus, bibliotecas e exposições.

Grupo 2 - Artes: este grupo inclui as indústrias criativas baseadas puramente em arte e cultura. A obra artística é inspirada no patrimônio, identidade de valores e no sentido simbólico. Este grupo é dividido em dois grandes subgrupos: a) artes visuais: pintura, escultura, fotografia e antiguidades; e b) artes performáticas: música ao vivo, teatro, dança, ópera, circo e marionetes. 
Grupo 3 - Mídia: este grupo abrange dois subgrupos de mídia que produzem conteúdo criativo com o objetivo de gerar comunicação com o grande público: a) publicações e mídia impressa: livros, imprensa e outras publicações; e b) audiovisual: cinema, televisão, rádio e outras formas de radiodifusão.

Grupo 4 - Criações funcionais: este grupo agrega atividades que são mais orientadas à demanda e atividades de criação de bens $\mathrm{e}$ serviços com fins funcionais. Está dividido nos seguintes subgrupos: a) design: interiores, gráfico, moda, joias e brinquedos; b) new media: software, games e conteúdo digital criativo; e c) serviços criativos: arquitetura, publicidade, P\&D, serviços digitais e outros serviços criativos relacionados.

Otro concepto que debe ser tratado en este apartado es el de "clase creativa". Se trata del grupo de profesionales, científicos y artistas que genera dinamismo económico, social y cultural, especialmente en áreas urbanas. La clase creativa también incluye a personas que forman parte de los campos de la ciencia e ingeniería, arquitectura y diseño, educación, artes, música y entretenimiento; es decir, cuya función económica es crear ideas, nuevas tecnologías y contenidos creativos. Estos profesionales comparten un acto creativo común, valorizando la creatividad, individualidad, diferencia y mérito, añadiendo a partir de eso, valor económico por medio de la creatividad. La estimación es que la clase creativa representaba aproximadamente el $30 \%$ de la fuerza de trabajo en los Estados Unidos y que el sector creativo contabilizaba casi la mitad de todos los ingresos y salarios en ese país, cerca de 1,7 billones de dólares ( Flórida 2012, p. 27).

Aún dentro de este capítulo se tiene el concepto de "ciudades creativas". Expresión que describe un complejo urbano en el que varios tipos de actividades culturales forman parte del complejo económico y social de la ciudad. Las ciudades creativas tienden a poseer una sólida infraestructura social y cultural, concentrando empleos creativos y, atrayendo inversión debido a sus facilidades culturales consolidadas.

Esto hace que la economía creativa tenga un impacto inestimable, especialmente en la generación de empleo. La economía creativa demanda conocimiento intensivo, necesitando habilidades específicas y alto nivel de calificación técnica de su mano de obra, demandando aún, trabajo intensivo, especialmente en sectores que poseen una alta concentración de insumos creativos, como por ejemplo: en las producciones teatrales y cinematográficas.

Según el informe del Instituto de Investigación Económica Aplicada, la economía creativa formal representa en Brasil entre el 1,2\% y el 2\% del PIB y, aproximadamente el $2 \%$ de la mano de obra, y el 2,5\% de la masa salarial formal, en el año 2010575 mil trabajadores formales (Araújo, et $a l, 2013$, p. 5). Es importante resaltar que estas informaciones son aproximadas; en cuanto que la economía creativa tiene una considerable proporción de agentes económicos en el mercado informal.

Actualmente la discusión de la economía creativa ( Leitão, 2014) gira alrededor de la creación de indicadores de rendimiento a fin de medir los resultados de la creatividad. En este punto los países europeos están más avanzados, debatiendo sobre el posible establecimiento de un Índice Europeo de Creatividad que se aplicará en la Unión Europea.

Esto demuestra que la economía creativa es una nueva manera de hacer economía, cuyo centro y producción de la riqueza ya no es la fábrica (economía de la chimenea), sino el conocimiento humano. Una vez que estamos ante una nueva forma de producción y distribución de riqueza surgen los desafíos y dudas conceptuales y teóricas sobre lo que es economía creativa. Se ha avanzado mucho en el reconocimiento y la clasificación de la economía creativa, pero aún queda mucho por hacer en términos de avance del conocimiento científico y la creación de políticas públicas adecuadas.

2.2. Aspectos teórico-conceptuales acerca de la Economía Social y Solidaria (ESS)

En Brasil y en muchos países, la ESS se viene presentando, en los últimos años, como innovadora alternativa de generación de trabajo y renta y una respuesta a favor de la inclusión socio laboral. Para muchos, puede ser considerada también como un nuevo modelo de desarrollo, más humano e inclusivo (Morais, et al, 2016). 
La ESS comprende una diversidad de prácticas económicas y sociales, que realizan actividades de producción de bienes, prestación de servicios, finanzas solidarias, intercambios, comercio justo y consumo solidario (Morais, 2013, 2014). Este fenómeno multifacético que progresivamente gana espacio en la academia, en las agendas de políticas públicas nacionales e internacionales y por la adhesión de sus actores, se constituye un área de estudio de gran importancia y pertinencia, además de gran interés y curiosidad para gran parte de la sociedad.

El campo de estudios y de praxis de la ESS contempla diferentes corrientes teóricas, que hoy influencian el pensamiento sobre su papel y lugar en la transformación del modo de producción capitalista. Es decir, este campo es caracterizado por la permanente tensión, sea en lo referente a cuestiones teórico-conceptuales, o entre las diferentes iniciativas locales, su escala, sus contextos, sus especificidades, sus encuadramientos y sus modos de operación.

Así, esta temática está impregnada por embates y conflictos que van desde la teoría a la praxis, ya que involucran diferentes posicionamientos en términos políticos, culturales, ideológicos y conceptuales. Si embargo, se cree que, como lo afirma Vieira (2005, p. 56), la ESS "enquanto conceito possui uma tripla natureza. Ao mesmo tempo em que é um objeto empiricamente verificável, também é um movimento social e uma teoria propositiva da mudança socioeconômica".

En esta perspectiva, Cruz (2006, p. 37) menciona que "é difícil precisar o momento em que o termo começa a ser utilizado, e vários teóricos reivindicam para si a primazia na utilização do conceito" (Montolio, 2002). Para este autor, conceptualizar estos "sectores" es algo complejo: "uma vez que é uma tarefa que se desloca do mero debate acadêmico, para incorporar-se aos olhares e propostas de um espectro amplo de atores sociais, do movimento sindical aos formuladores de políticas públicas, de instituições religiosas a organismos multilaterais".

En la literatura económica occidental, se pueden detectar tres enfoques teóricos bien definidos: a) enfoque de la Economía Social, considerado como el más antiguo, más consolidado y bastante divulgado, frente a estas dificultades, de acuerdo con Moreno y Chaves
(2006) y utilizado en Europa; b) enfoque de las organizaciones "Non Profit" y; c) enfoque de la Economía Solidaria, el más reciente y utilizado en América Latina y en Brasil (Morais, 2013).

La diversidad terminológica existente fue minuciosamente descrita por Moreno (1996) y, de acuerdo con la autora, los términos más frecuentes son: tercer sector; sector voluntario; sector no lucrativo; sector filantrópico; sector caritativo; organizaciones no gubernamentales (ONG); sector independiente; sector intermedio; sector exento de impuestos; economía social y economía solidaria. Tremblay (2009) también enumera, por países, diferentes terminologías, como Social Economy (Estados Unidos y Canadá), Economía Solidaria (Argentina, Brasil, Chile y Quebec), Economía Popular (Asia), Associative Movements (Senegal y Turquía), “Civil Society” (África del Sur) y Community Economic Development (Australia, Nueva Zelanda y Canadá anglosajón).

Estudio reciente realizado por la Inter-Agency Task Force on Social and Solidarity Economy (UNRISD, 2018) en el que la fuerza de trabajo de la ESS en el marco de las Naciones Unidas, asigna diferentes terminologías utilizadas en documentos y publicaciones oficiales (de gobiernos) en todos los continentes. Se entiende que tal mapeamiento refuerza la idea de que la gran cantidad de terminologías está justificada por los diferentes modos de generación, actuación y comportamiento que este sector manifiesta en los diferentes países, es decir, en cada nación, así como en cada región, adoptan distintas terminologías para hacer frente al sector, de acuerdo con aspectos históricos, culturales, políticos, jurídicos, etc. "La elección de un objeto como objeto de estudio por parte del científico social evidencia la existencia de juicios de valor en el proceso científico y denota una indudable carga política e ideológica, que, como señalan Moreno y Chaves" (Moreno y Chaves, 2006, p. 91).

\section{ESS}

\subsection{Elementos característicos de la}

Por falta de consenso para definir, clasificar, medir y sistematizar el campo de la ESS y qué organizaciones forman parte, es necesario presentar algunos elementos característicos que académicos, profesionales y políticos vienen utilizando para identificar estas organizaciones, entidades y empresas. En ese sentido, al detectar tales elementos, es posible también diferenciar 
tales organizaciones y emprendimientos de las "tradicionales".

El rasgo característico inicial es la premisa de que producen bienes o servicios con valor social. En otras palabras, el Consejo Mundial de Cooperativas de Crédito resume en: "no por el lucro, es por la caridad, campaña por el servicio". Así, el beneficio es esencial para la sostenibilidad y el desarrollo de las organizaciones y empresas de la ESS. Sin embargo, no se considera como meta final, además de que se utiliza y distribuye según las reglas específicas inherentes a las estructuras jurídicas previamente acordadas y definidas. El beneficio y el excedente productivo son necesarios para la viabilidad socioeconómica de los EES y son, generalmente utilizados para la ampliación de los negocios y perfeccionamiento de los recursos humanos y de la infraestructura de los actores involucrados.

Para Fontaneau, et al. (2010), otras características, además de la función económico-social, pueden ayudarnos a identificar organizaciones y empresas de la ESS. Son ellas: participación, solidaridad, participación voluntaria, autonomía y dimensión colectiva. Como ejemplos de organizaciones y emprendimientos asociados a la ESS, se citan: cooperativas, las $\mathrm{ONG}$, fundaciones, institutos, asociaciones, empresas sociales, sociedades mutuas, emprendimientos económicos solidarios (EES, en el caso específico de Brasil). Todas ellas pasibles de amplia discusión acerca de su efectividad en cuanto ESS.

En términos generales, a partir de Fonteneau et al (2010), así como del amplio debate internacional que realizamos en el marco de la OIT, se puede decir que:

a) La ESS se refiere a formas específicas de organizaciones y empresas, siendo los tipos más comunes, las cooperativas, las sociedades mutuas, las asociaciones, las organizaciones comunitarias, las empresas sociales, las fundaciones. Se constituye, por lo tanto, en grupo de organizaciones dinámicas y en evolución;

b) Las organizaciones de ESS poseen características comunes que las diferencian de organizaciones de la economía pública y de la economía privada tradicional, pues comparan principios operativos específicos basados en la participación, la solidaridad, la innovación, la incorporación voluntaria y la propiedad colectiva;

c) La amplitud existente de denominaciones son conceptos divergentes, pero relacionados. Todos poseen ciertos orígenes geográficos y antecedentes teóricos que enfatizan dimensiones particulares de esta forma económica;

d) Las organizaciones de ESS pueden ofrecer ventajas comparativas para abordar los retos sociales, económicos y políticos de todo el mundo, incluida la cohesión social, el empoderamiento y el reconocimiento de una economía plural;

e) La ESS está adquiriendo más importancia en el momento actual, teniendo en cuenta la crisis económica global, ya que las salidas exigirán, entre otros aspectos, un modelo de desarrollo más inclusivo y sostenible (Morais, Dash y Bacic 2016).

En esta perspectiva, Rodriguéz (2018, p. 6) bien sintetiza la ESS como un conjunto de prácticas socioeconómicas que "combinan la empresarialidad cooperativa con la asociación de personas en busca de satisfacer necesidades".

\section{ENCONTRANDO CONEXIONES ENTRE LA ECONOMÍA CREATIVA Y LA ECONOMÍA SOCIAL Y SOLIDARIA}

La economía creativa no es solo un conjunto de sectores encuadrados en una nueva categoría, sino el emblema de un nuevo ciclo económico, como respuesta a los renuentes problemas globales, motivando y creando nuevos modelos de negocios, procesos organizacionales y relaciones institucionales entre los agentes económicos y sociales, las cuales no se miden adecuadamente, hasta el momento.

La economía creativa surge en el escenario de desmaterialización de las relaciones de trabajo y de formación de una economía basada en la información y el conocimiento, así como en un contexto de alta dinamización de la cultura de consumo y de estilos de vida alternativos al modelo fordista, alejando aún la antigua visión negativa de la "Industria de la Cultura" creada por la Escuela de Frankfurt en la que la cultura era un mecanismo de homogeneización del pensamiento social. 
En este nuevo paradigma, que incluye la cultura en su esencia y la tecnología como un medio de propulsión, la organización de los mercados en redes, alianzas entre agentes económicos y sociales, la prevalencia de aspectos intangibles de la producción, utilización de nuevas tecnologías para la producción, distribución y/o acceso a bienes y servicios y la singularidad de la producción, fuertemente anclada en la singularidad, son características de este modelo que es supuestamente sustentable, mejorar el bienestar y la inclusión socioeconómica, superando así el llamado "paradigma de la chimenea".

La ejecución de este modelo permite la integración vertical ofreciendo a individuos y colectividades la posibilidad de un desarrollo ambientalmente equilibrado dada la conexión entre la creatividad, la condición humana y la cuestión territorial, que en verdad, tiene naturaleza ambiental.

El éxito (efectividad y eficiencia) de la economía creativa depende necesariamente de la colaboración entre los agentes económicos, ya sean productos o consumidores. El conjunto de reglas más avanzado para promover esta interacción (que también involucra a la sociedad) es la Economía Social y Solidaria.

Aunque no hay una fórmula para el éxito, la economía creativa parece ser, de hecho, un importante potencial de desarrollo socioeconómico, aprovechando un momento de transición de paradigmas globales para reorganizar los recursos y la distribución de beneficios económicos. Pero, como hemos visto, aunque la creatividad como un recurso omnipresente reconocido, es necesario percibir su potencial económico, sobre la base de once puntos de motivación:

1) discutir las bases filosóficas y sociológicas de los cambios causados por la revolución tecnológica y sus impactos en la sociedad, el medio ambiente y el derecho;

2) la conciencia que la inclusión se hace por convergencia de intereses;

3) definir y aplicar políticas de desarrollo;

4) influir en los acuerdos internacionales de modo que permitan la apropiación de los beneficios de la economía creativa por las comunidades que originaron;
5) promover el acceso adecuado a la financiación;

6) recoger datos estadísticos que acompañan el desarrollo de acciones de políticas públicas; 7) proporcionar suficiente tecnología e infraestructura de comunicación;

8) establecer un modelo de gobernanza coherente;

9) analizar el proceso de generación de valor no en una estructura de cadena, sino en las redes;

10) garantizar nuevos profesionales adecuados y de nuevas profesiones;

11) forman un ambiente que reconoce el valor económico de la creatividad y del patrimonio cultural inmaterial.

Esto abre oportunidades en dos frentes: una, resultante del proceso de desindustrialización de las grandes ciudades brasileñas que lleva a la búsqueda de actividades económicas sustitutivas; y otra, que analiza el impacto de la industria cultural y de la clase creativa en la economía regional y nacional, como una forma de desarrollo económico y social.

Como los cambios de paradigmas pueden traer oportunidades, la pérdida de esas oportunidades genera un costo - social, cultural, económico. Somos responsables, por tanto, de decidir si queremos crear las condiciones para que la diversidad y los talentos creativos de los países en desarrollo se convierten en un activo económico o se opongan a perpetuarse las disparidades históricas en que se vive hoy.

Es claro que los cambios tecnológicos insertados en un marco de búsqueda frenética por la acumulación de capital como objetivo último no serán capaces de contribuir al enfrentamiento del cuadro de desigualdad, exclusión, desempleo, etc. En otros términos, el cambio de paradigma tecnológico debe estar acompañado por el cambio de paradigma en relación al modelo de desarrollo que se busca. En esta perspectiva, la ESS se sitúa como un instrumento pertinente para reflexionar y actuar en pro de este nuevo escenario y los desafíos inherentes.

Como se mostró, la ESS contempla elementos que combinan la generación de trabajo y renta con la participación social y comunitaria, así como la preservación del medio ambiente, la inserción socioeconómica de los involucrados 
y la preocupación con el entorno territorial. Es decir, abarca factores de orden económico, pero también social y ambiental.

En este sentido, ya hay algunas experiencias que conectan la ESS y la economía creativa. Tales experiencias pueden encuadrarse en lo que se sugiere acuñar como "tecnologías sociales". Sobre el término, no existe una definición exacta y consensuada, sin embargo, se desprende que el concepto Tecnología Social advenga de Brasil (Duque y Valadão; 2017). El Instituto de Tecnologia Social (ITS), por ejemplo, entiende las tecnologías sociales como técnicas y metodologías reaplicables, desarrolladas $\mathrm{y} / \mathrm{o}$ aplicadas en la interacción con la población, , casi siempre, representando soluciones para la inclusión social y la sustentabilidad ambiental, llevando la mejora de las condiciones de vida.

Otra referencia importante en Brasil es la Fundación Banco do Brasil (FBB) que desde hace algunos años estimula las Tecnologías Sociales a partir del Banco de Tecnologías Sociales y de la premiación anual. Este estímulo es importante para la diseminación y el fortalecimiento de las tecnologías sociales, y para permitir una mayor sensibilización de la sociedad basada en experiencias locales reales, en diferentes áreas como energías renovables, uso del agua, educación, seguridad alimentaria, residuos sólidos, etc.

De manera general, se entienden las Tecnologías Sociales como una visión alternativa a los fines eminentemente economicistas de las tecnologías convencionales, así como forma de ir hacia debate más complejo acerca de la necesidad de pensar otro modelo de desarrollo socioeconómico, para mezclar la inclusión social a los avances tecnológicos de esta nueva era. En este "otro" modelo, gobiernos, instituciones de enseñanza y de investigación, empresas, agencias de financiamiento y la sociedad civil, deben estar en permanente diálogo para el diseño, la implementación y la evaluación de los proyectos que involucran las tecnologías sociales.

Es decir, es necesaria una nueva cultura institucional que sea favorable a las Tecnologías Sociales; una cultura que contemple la construcción de una agenda para el tema, para conectar los avances tecnológicos con el contexto social, político, humano y ambiental, así como sus demandas. No se trata de una simple adaptación de la tecnología al campo de la innovación social, sino de realizar un enorme giro conceptual que tenga en cuenta la satisfacción de otro conjunto de valores e intereses coherente con el modelo de desarrollo que se desea crear.

Como ejemplos ya en boga, se citan los bancos comunitarios y las monedas sociales, las plataformas on line de comercialización solidaria, además de otras innumerables tecnologías sociales (que pueden ser accedidas, de forma sistematizada, en el banco de datos de tecnologías sociales de la Fundación Banco do Brasil).

De forma panorámica, es posible registrar la existencia de experiencias, en los ámbitos nacional e internacional. Son experiencias que, a menudo, cargan consigo fragilidades estructurales inmanentes, pero que apuntan a potencialidades de transformación de muchos territorios. Con base en Morais (2017), se mencionan:

a) Estrategias de convivencia con el semiárido brasileño con el fortalecimiento de la agricultura familiar a partir de proyectos de represa para captación de agua;

b) la energía alternativa renovable y gratuita para la inmigración y el cultivo;

c) Montaje de biodigestores en escuelas públicas que sustituyen al gas de cocina;

d) Proyectos arquitectónicos para minimizar el déficit habitacional de personas de bajos ingresos;

e) plantas de biodiesel de bajo costo, con reciclaje del aceite de fritura usado para abastecer barcos de pesca artesanal;

f) Proyectos de cultura digital con valorización de la cultura popular;

g) Experiencias diversas en el área de la economía solidaria, como las monedas sociales, plataformas digitales (redes), bancos comunitarios, etc.

h) Además de innumerables experiencias más recientes que, reconocemos, carecen de una mirada más atenta, como el "mundo de las iniciativas de ciberciencia ciudadana", en proyectos y acciones de "crowdsourcing científico", así como de movimientos tales como Media Labs, Hack Labs y Fab Labs, etc.

Además, desde el punto de vista de la inserción del tema en la política pública, es interesante mencionar el caso de la política pública municipal 
de ESS que, se alió con la economía creativa, en la ciudad de Araraquara, interior del Estado de São Paulo, dando lugar a la Coordinadora Ejecutiva de Trabajo, Economía creativa y Solidaria; además de la realización de la $1^{\text {a }}$ Conferencia Municipal de Economía Creativa y Solidaria y la creación del Sistema Municipal de Economía creativa y Solidaria. Así, la política pública de economía creativa y solidaria pretende fortalecer EES y sus redes de cooperación en cadenas de producción, comercialización y consumo, contando con acciones ligadas a la formación, fomento, crédito y comercialización, representando la ampliación de las oportunidades de inserción productiva, a través del trabajo, de segmentos excluidos del mercado formal y de personas que buscan actuar en otra lógica de desarrollo, donde el centro es el ser humano en su totalidad, más solidario, con justicia social, y que no agrada el medio ambiente.

Por lo tanto, es estratégico para ampliar y fortalecer la cultura y las estrategias de fomento de la economía creativa y solidaria, ver en ésta, una forma de generación de ingresos, organización, producción y relación social, más justa, equitativa, en las dimensiones humana, social, cultural, política y económica, de forma tal que este modo de producción se presente como una verdadera opción de organización económica, de proyecto para la sociedad y que tenga un papel destacado y prioritario entre las políticas públicas de desarrollo sustentable, estimuladas por el Gobierno Municipal.

Esto muestra que la generación local de ingresos, a partir de instrumentos de la ESS y de la economía creativa, constituye uno de los elementos que puede contribuir al desarrollo local-territorial, ya que genera dinámica económica, sobre todo en regiones más periféricas, con bajo dinamismo económico y alto índice de pobreza. Se trata, pues, del desarrollo endógeno, con base en la visión de que los sistemas productivos consisten en un conjunto de factores materiales e inmateriales que permiten que las economías locales y regionales adopten caminos diferentes para el crecimiento económico y el desarrollo social.

Unificar las posibilidades abiertas por la economía creativa con las potencialidades de la ESS es el actual desafío -teórico y práctico-. Si las estructuras económicas y sociales cambian a partir de los modos de producción y consumo provenientes de la economía creativa, la estructura jurídica también debe cambiar para acompañar las nuevas estructuras.

En este punto, las estructuras jurídicas son el principal punto de cambio que debe ocurrir para el avance de la economía creativa. Hoy, en todo el mundo, se debate cómo debe regularse la economía creativa, y esta regulación puede nacer con los principios de la economía solidaria ya incorporados.

El Derecho aún no comprende lo que es economía creativa, habiendo una fuerte duda en el medio jurídico de cómo clasificar las actividades de economía creativa (Siqueira N., y Menezes, 2017). En la ESS tampoco existe una adecuada comprensión de lo que son los ESS, dificultando o incluso impedimento, la legalización de estos emprendimientos.

Uno de los caminos, quizá sea retomar la antigua discusión de la Economía del Bien Común que apunta a un nuevo concepto -sociológico y jurídico- acerca de la propiedad. Se resalta -sobre todo a los desavisados- que la economía del bien común no es el retorno al comunismo de Estado, sino una contestación al capitalismo, surgida en la década del 90 a partir de los movimientos ecológicos globales ( Dardot y Laval, 2017). Lo que caracteriza al "común" es el uso, o sea, una utilidad común, lo que crea una obligatoriedad pública de mantener el bien común, creando una fuerza colectiva que no es sólo económica, sino social y ambiental.

A partir de la obra de Elinor Ostrón, Dardot y Laval (2017) proponen una nueva clasificación de la propiedad: 1) bienes puramente privados (rivales y exclusivos); 2) bienes puramente públicos (no rivales y no exclusivos); 3) bienes de club (exclusivos y no rivales - consumo es individual, pero no es disminuido por el consumo de otras personas -por ejemplo, espectáculo artístico); 4) bienes comunes -los common goods (no exclusivos y rivales- a los cuales no se pueden restringir el acceso, pero la explotación individual puede llevar a su agotamiento, por ejemplo, las zonas de pesca). El modelo de bien común es interdisciplinario y aplicable a todos los tipos de empresas y organizaciones y puede ser considerado como la herramienta que necesita ser comprendida y reanudada para que se establezca 
la conexión necesaria entre economía creativa y la ESS.

\section{CONCLUSIONES}

Como se intentó mostrar, se vive actualmente, en una nueva era, marcada por profundas e irreversibles cambios tecnológicos cuyos desdoblamientos todavía son desconocidos. Esta nueva era se caracteriza por avances que van hacia la inteligencia artificial, internet de las cosas, big data, computación en nube, vehículos autónomos, robótica avanzada, etc.

Sin embargo, por otro lado, se percibe que los cambios tecnológicos se insertan en un cuadro de búsqueda frenética de la acumulación de capital y no pretenden contribuir, definitivamente, al enfrentamiento de la desigualdad, exclusión, desempleo, etc. Así, se entiende que el cambio de paradigma tecnológico debe estar acompañado por el cambio de paradigma en relación al modelo de desarrollo que se busca.

Se inserta entonces la pertinencia y la relevancia en discutir las conexiones entre la economía creativa y la ESS. Hasta, porque no toda economía creativa es solidaria y no toda ESS es creativa.

La economía creativa puede ser entendida como una nueva manera de hacer economía, ya que estamos ante una nueva forma de producción y de distribución de riqueza. En esta dirección, la economía creativa parece ser, de hecho, un importante potencial de desarrollo socioeconómico, aprovechando un momento de transición de paradigmas globales para reorganizar los recursos y la distribución de los beneficios económicos.

En esta perspectiva, la ESS se sitúa como instrumento para reflexionar y actuar en pro de este nuevo escenario y los desafíos inherentes. La ESS contempla elementos que combinan la generación de trabajo y renta con la participación social y comunitaria, así como la preservación del medio ambiente, la inserción socioeconómica de los involucrados y la preocupación con el entorno territorial. Es decir, abarca factores de orden económico, pero también social y ambiental.

Como ejemplos prácticos, ya en marcha, se presentaron las experiencias de las tecnologías sociales que, aunque no tienen una definición precisa, apuntan a contribuciones reales y efectivas, con desdoblamientos fértiles en los territorios de actuación.

Una estrategia inmediata es fortalecer las políticas públicas a través de leyes locales que integren los principios de la economía solidaria a la economía creativa. Sin embargo, todavía hay mucho que hacer en términos de avance del conocimiento científico y de la creación de políticas públicas adecuadas en el marco de la economía creativa, sea de la ESS. Adicionalmente, se entiende que en este debate es adecuado retomar la discusión del bien común y sus desdoblamientos y contribuciones como un eslabón entre la economía creativa y la ESS.

\section{BIBLIOGRAFÍA}

- Araújo, B. C.; Oliveira, J. M. de \& Silva, L. V. (2013). Panorama da Economia criativa no Brasil. Texto para Discussão 1.880. Rio de Janeiro: IPEA.

- Cruz, A. C. (2006). A diferença da igualdade: a dinâmica da economia solidária em quatro cidades do Mercosul. (Tesis doctoral), Universidade Estadual de Campinas, Campinas, Brasil.

- Dardot, P. \& Laval, C. (2017). Comum Ensaios sobre a Revolução no Século XXI. São Paulo, Boitempo.
- Duque, T. \& Valadão, J. “Abordagens teóricas de tecnologia social no Brasil". Revista Pensamento Contemporâneo em Administração. 11(5), (out-dez de 2017).

- Flórida, R. (2012). The Rise of the creative class. New York: Basic Books.

- FIRJAN - Federação das Indústrias do Rio de Janeiro (2019). Mapeamento da Indústria Criativa no Brasil. Disponible en https:// www.firjan.com.br/EconomiaCriativa/ downloads/MapeamentoIndustriaCriativa. pdf 
- Fonseca, R. \& Machado, L. (2013). "Indicadores de Penetração e Uso da Internet por Empreendimentos da Economia Solidária". Revista Tecnologia e Sociedade. 9(17), pp. 54-65,

- Fontaneau, B.; Neamtan, N. N.; Wanyama, F.; Morais, L. \& Poorter, M. (2010). "Social and Solidarity Economy: building a common concept". 1st. International Academy of SSE. ITCILO: Turin.

- Jaramillo, M. (2005). Manual de cooperativismo y economía solidaria. Bogotá: Universidad Cooperativa de Colombia.

- Leitão, C. (s. f.) Plano da Secretaria da Economia criativa: políticas, diretrizes $e$ ações 2011-2014. Disponible en http:// observatoriodadiversidade.org.br/ site/\%E2\%80\%9Csomos-um-paiscriativonao-um-pais-inovador\%E2\%80\%9D-dizclaudia-leitao/.

- Lisboa, A. (2017) "Economia compartilhada e economia solidária: interfaces, continuidades e descontinuidades". Revista NECAT. 6(11), pp. 8-32.

- Montolio, J. M. (2002). “Economía Social: concepto, contenido y significación en España". Revista CIRIEC-España, No. 42, pp. 05-31.

- Morais, L. P. (2013). As políticas públicas de Economia Solidária (ESOL): avanços e limites para a inserção sociolaboral dos gruposproblema. (Tesis doctoral), Universidade Estadual de Campinas, Campinas, Brasil.

- Morais, L. P. (2014). Social and Solidarity Economy and South-South and Triangular Cooperation in Latin America and the Caribbean: Contributions to Inclusive Sustainable Development. Géneve, ILO. Disponible en http://socialeconomy.itcilo. org/en/readers

- Morais, L. P..; Aash, A. \& Bacic, M. J. (2016). "Social and Solidarity Economy in India and Brazil". 2016 Social Enterprise Summit \& 4th International Conference on Social Enterprise in Asia. Hong Kong.
- Morais, L. P. (2017). "Ciência, tecnologia e inovação (CT\&I) para o desenvolvimento inclusivo e sustentável: breves reflexões sobre o papel das tecnologias sociais". Radar Ipea, No. 54, pp. 19-22.

- Moreno, A. S. (1996). Análisis económico del sector no lucrativo. València: Tirant lo Blanc.

- Moreno, A. S. \& Chaves, R. (2006). "Balance y tendencia en la investigación sobre tercer sector no lucrativo. Especial referencia al caso español". Revista CIRIEC-España, No. 56, pp. 87-116.

- Peruffo, E.; Schmidlechner, R.; Contreras, R. \& Molinuevo, D. (2017). "Automation, digitisation and platforms: implications for work and employment. Concept Paper". En European Foundation for the Improvement of Living and Working Conditions. Dublin.

- Rodriguéz, J. F. (2018). Economía social y solidaria en el territorio: significantes y coconstrucción de políticas públicas. Bogotá: Fundación Cultural Javeriana.

- Siqueira Neto, J. F. \& Menezes, D. F. N (2017) Conceptos de Economía Solidaria en América Latina - Discusión de Economía creativa. Dusseldorf: Editorial Académica Española.

- Tremblay, C. (2009). "Advancing the social economy for socio-economic development: international perspectives". Canadian Social Economy Research Partnerships Public Policy Papers Series, No. 1.

- UNCTAD. (2010) Relatório Economia criativa: Uma Opção de Desenvolvimento Viável. Disponible en http://unctad.org/pt/ docs/ditctab20103_pt.pdf

- UNCTAD. United Nations Conference on Trade and Development (2018). Creative economy Outlook Trends in international trade in creative industries 2002-2015. Country profiles 2005-2014. Genebra.

- Vieira, F. M. (2005). Coerência e aderência da economia solidária: um estudo de caso dos coletivos de produção do MST em Mato Grosso do Sul. (Tesis doctoral). Universidade de São Paulo, São Paulo, Brasil. 